\title{
Characterization of headspace volatile flavor compounds formed during kefir production : application of solid phase microextraction.
}

\begin{abstract}
Headspace volatile flavor compounds of kefir were monitored using headspace solid phase microextraction (HS-SPME) method during fermentation of milk with kefir starter culture. Among all flavor compounds, forty volatile compounds were initially detected using gas chromatography coupled to time-of-flight mass spectrometer (GC-TOFMS). Consequently, eight volatile flavor compounds, namely ethanol, ethyl acetate, ethyl butyrate, 2-butanone, acetone, 3-hydroxy-2-butanone (acetoin), 2,3-butanedione (diacetyl) and acetaldehyde were considered as the representative of the alcohol, ketone, ester and aldehyde compounds in kefir. Moreover, in term of quantitative analysis, more than $97 \%$ of total flavor compounds composed of the proposed volatile flavor compounds. The results indicated that the concentration of 2-butanone released into headspace of kefir was found to be stable during fermentation. The release content of other volatile flavor compounds increased throughout the fermentation process. However, the headspace concentration of acetoin significantly $(\mathrm{P}<$ 0.05 ) decreased between $\mathrm{pH} 5.2$ and 4.6.
\end{abstract}

Keyword: Volatile flavor compounds; Kefir; Headspace solid phase microextraction; Fermented milk. 